\title{
Local Growth of CuInSe2 Micro Solar Cells for Concentrator Application
}

Berit Heidmann $^{\dagger, a, b}$, Franziska Ringleb ${ }^{c}$, Katharina Eylers ${ }^{c}$, Sergiu Levcenco ${ }^{\mathrm{d}}$, Jörn Bonse ${ }^{\mathrm{e}}$, Stefan Andree , Jörg Krüger ${ }^{\mathrm{e}}$, Thomas Unold ${ }^{\mathrm{d}}$, Torsten Boeck ${ }^{\mathrm{c}}$, Martha Ch. Lux-Steiner ${ }^{\mathrm{a}}$, Martina Schmid ${ }^{\mathrm{a}, \mathrm{b}}$

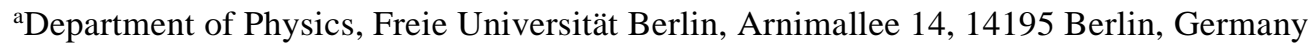

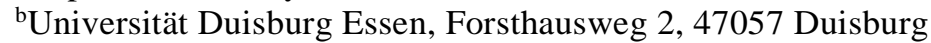 \\ ${ }^{c}$ Leibniz Institute for Crystal Growth, Max-Born-Straße 2, 12489 Berlin, Germany \\ ${ }^{\mathrm{d} S}$ Struktur und Dynamik von Energiematerialien, Helmholtz-Zentrum Berlin, Hahn-Meitner-Platz 1, 14109 \\ Berlin, Germany \\ ${ }^{\mathrm{e}}$ Bundesanstalt für Materialforschung und -prüfung (BAM), Unter den Eichen 87, 12205 Berlin, Germany
}

\section{$\dagger$ Corresponding author: berit.heidmann@helmholtz-berlin.de}

Keywords: micro solar cells, light concentration, $\mathrm{CuInSe}_{2}$, absorber optimization, chalcopyrite

\begin{abstract}
A procedure to fabricate $\mathrm{CuInSe}_{2}$ (CISe) micro-absorbers and solar cells for concentrator applications is presented. The micro-absorbers are developed from indium precursor islands which are deposited on a molybdenum coated glass substrate (back contact), followed by deposition of copper on top and subsequent selenization as well as selective etching of copper selenides. In order to compare the properties of the locally grown absorbers to those of conventional large area CISe films, we systematically examine the compositional and morphological homogeneity of the micro absorbers and carry out photoluminescence measurements. Preliminary devices for micro-concentrator solar cell applications are fabricated by optimizing the copper to indium ratio and the size of the indium precursor islands. The resulting micro solar cells provide a characteristic $I-V$ curve under standard illumination conditions (1 sun).
\end{abstract}

\section{Introduction}

Renewable energies are becoming an important pillar of power generation in the last decades. Photovoltaics constitute a significant contribution in this sector, but still cost reduction remains one of the key challenges for competitiveness to fossil sources [1].

Concentrator photovoltaic (CPV) systems, which represent an alternative design to classical flat panels, offer a possibility to increase the solar cell efficiency. However, due to their bulky configuration, they are currently not easily applicable for roof-top application since they work with direct light and need a tracking system, which is not possible for fixed installations. Commercially available classical CPV modules focus the sunlight onto the active cell area, which is typically in the range of $10 \mathrm{~cm}^{2}$ and work in the high concentration regime, i.e. with a concentration factor $>300$ [2]. The 
higher the concentration, the more critical becomes heat accumulation. The latter reduces the cell efficiency and lifetime and must be addressed by dissipation techniques like active cooling. In short, CPV systems allow increasing cell efficiencies, but have disadvantages compared to conventional PV regarding the scope of application and costs.

Micro-concentrator photovoltaic devices with an active area of up to $\sim 1200 \mu \mathrm{m}^{2}$ per cell and small micro-optics can overcome the problems of heat accumulation and bulkiness by a better heat dissipation [3] and a low profile module design. Furthermore, the approach lowers costs for lenses and tracking systems, due to a reduced mass and size of the system. Thus, this approach promises the opportunity to access the high-performance/low-cost region between the conventional CPV and flatplate (1 sun) PV domain, while widening the range of applications [4] and allowing simultaneous material saving. Theoretically, an efficiency enhancement of about $12 \%$ absolute can be achieved for maximum concentration [5]. In top-down approaches micro solar cells are fabricated for example by lithography from planar absorbers. Here the efficiency potential of concentrator micro-absorbers has already been demonstrated for silicon [6] as well as for chalcopyrite solar cells [3,7-9], where an absolute efficiency enhancement of 5\% has been reached [9]. Furthermore, a first mini-module based on chalcopyrite absorbers showed an absolute efficiency gain of 1.8\% [10].Even though the potential of efficiency increase has been demonstrated by these micro CPV studies, material saving is another concerning factor, particularly for chalcopyritebased systems, since here, costly constituents such as indium are required.

A possibility for simultaneous efficiency enhancement and material saving can be achieved by growing the micro-absorbers in a bottom-up process, i.e. the local manufacturing of solar absorbers at sites predefined by the geometry of concentrator optics - ideally an array of micro-lenses. Such an approach has been recently demonstrated using electrodeposition, and achieving a proof-of-concept solar cell consisting of micro dots with an efficiency of around $0.3 \%$ [11]. Using a similar approach 105 and $1105 \mu \mathrm{m}$ wide line shaped cells were realized exhibiting efficiencies of $5.3 \%$ and $7.6 \%$, respectively [12].

Alternatively, we propose a new bottom-up strategy for material saving in microconcentrator solar cells based on well-known thin film chalcopyrite absorbers. Such thin film materials can be deposited as localized islands allowing a site-controlled 
growth of the solar absorber and a production towards micro solar cell arrays. Since the final micro cells must be aligned to a micro lens array, the indium precursor islands must be regularly arranged and exhibit a suitable morphology. In a previous publication we have recently demonstrated that indium islands as precursor material can be site-selectively grown on the back contact (a molybdenum layer deposited on glass) taking benefit of the fs-laser processing technology [13,14]. This allows to arrange the precursor material into a regular array matching the lateral dimensions of micro-lens arrays.

In this study, we develop a procedure to transform such indium precursors to chalcopyrite micro-absorbers. Based on a sequential process well-established for the production of standard $\mathrm{CuInSe}_{2}$ chalcopyrite (CISe) cells, we transfer this technology into a bottom-up approach and demonstrate the manufacturing of micro-solar cells. Although the significantly different surface to volume ratio of the micro absorbers could be expected to result in deteriorated structural and optoelectrical properties, we observe characteristics comparable to planar absorbers.

\section{Experimental Section}

The fabrication of the micro solar cells is divided into two steps: In a first step, microabsorbers were grown and optimized in morphology, composition and optical homogeneity. In a second step, individual micro-absorbers were selected and manufactured into micro-solar cells.

\subsection{Fabrication of micro-absorbers}

a)

b)

c) d)

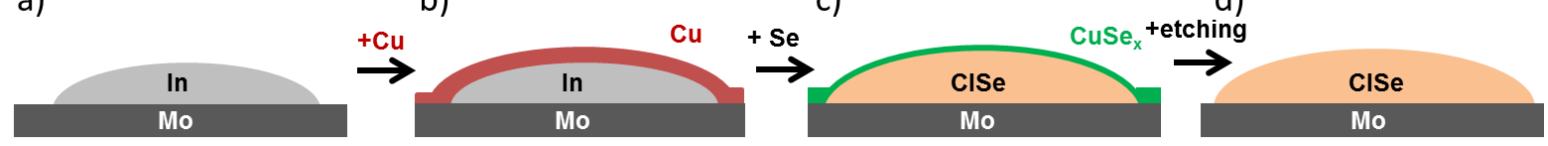

Figure 1: Scheme of bottom-up process for preparation of ClSe micro-absorbers. a) Bare indium island on molybdenum covered substrate, b) indium island overcoated with a flat copper layer, c) sample after selenization process, d) CISe absorber after etching of $\mathrm{CuSe}_{\mathrm{x}}$.

Figure 1 schematically shows the manufacturing process of our CISe micro-absorbers. Locally grown indium precursors on molybdenum coated glass were prepared by PVD at a substrate temperature of $510^{\circ} \mathrm{C}$, which resulted in the growth of indium islands (Figure 1a). The total nominal indium thickness (film equivalent) was $100 \mathrm{~nm}$. The indium island size was adjusted by the indium deposition rate. Small islands (diameter: $40 \mu \mathrm{m}$ ) were obtained by a deposition rate of $\sim 0.5 \AA / \mathrm{s}$, large islands (diameter: $60 \mu \mathrm{m}$ ) resulted from a deposition at 
$\sim 0.3 \AA$ /s (for more details of optimizing the indium island self-assembled growth, refer to our previous work $[13,14])$. After cooling to room temperature, a copper layer of several hundred nanometer thickness was deposited at a nominal rate of $1 \AA$ /s by PVD to obtain the desired indium-copper precursors (Figure 1b). For optimization, the $\mathrm{Cu}$ film thicknesses were varied in the range between 150 and $1000 \mathrm{~nm}$, depending on the indium island size. The precursor was converted to CISe in a selenization process consisting of two different temperature steps (Figure 1c). The sample was placed in a graphite box along with $0.046 \mathrm{~g}$ elemental selenium shots. In the first step (alloying phase), the whole box was heated up to $200^{\circ} \mathrm{C}$ and kept at this temperature for $10 \mathrm{~min}$. As the temperature is not high enough for selenium to be integrated into the metal precursor, a Cu-In alloy forms. This alloying phase is essential for the further reaction kinetics and phase formation of the absorber [15]. In the second step the selenization takes place by rising the temperature to $550^{\circ} \mathrm{C}$ and holding for $6 \mathrm{~min}$. The selenization was carried out in nitrogen atmosphere at 800 mbar pressure to avoid oxidation reactions with the ambient atmosphere. Local CISe absorbers were formed as well as a thin cover layer of copper selenides $\left(\mathrm{CuSe}_{\mathrm{x}}\right)$, which were selectively removed by a chemical wet-etching step in a $10 \% \mathrm{KCN}$ solution for 3 min (Figure 1d).

\subsection{Fabrication of micro solar cells}

In order to fabricate solar cells from the micro-absorbers locally grown on the molybdenum back contact, an additional electrical isolation is required. Therefore, an isolation layer of SU-8 photoresist [16], was spin coated on the sample (Figure 2a) and subsequently pre-baked at $100^{\circ} \mathrm{C}$, exposed to UV-light with a wavelength of $385 \mathrm{~nm}$ and hard-baked at $200^{\circ} \mathrm{C}$. As a result of the spin coating process, the final isolation layer on top of the micro-absorbers is significantly thinner than in between them. This allowed to remove the thin isolating capping layer on the micro-absorbers in an additional $\mathrm{Ar}^{+}$plasma etching process (Figure 2b).

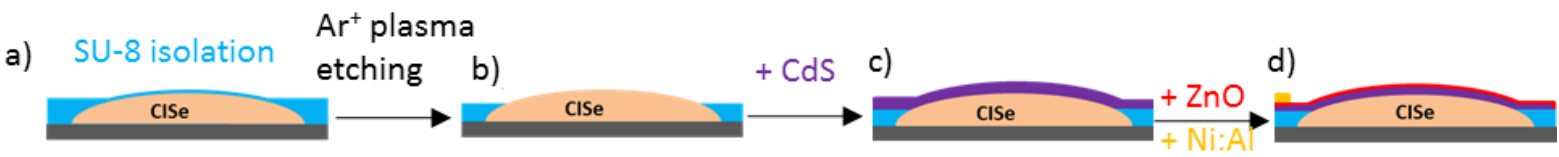

Figure 2: Scheme of electrical isolation process of the micro-absorber, consisting of spin-coating of SU-8 (a), $\mathrm{Ar}^{+}$plasma etching (b), CdS buffer layer deposition (c), and ZnO front contact deposition (d).

As a result, the micro-absorbers protrude (electrically non-isolated) above the residual isolation layer, which allows to contact them with a transparent window layer, consisting of a $\mathrm{CdS}$ buffer (Figure 2c) and a $\mathrm{ZnO}$ front contact (Figure 2d). For that, a $50 \mathrm{~nm}$ thick $\mathrm{CdS}$ buffer layer was deposited by chemical bath deposition in $1.1 \mathrm{~mol}$ ammonia, $0.14 \mathrm{~mol}$ thiourea and $0.002 \mathrm{~mol}$ cadmium acetate solution, heated to $60^{\circ} \mathrm{C}$. A $380 \mathrm{~nm} \mathrm{ZnO} / \mathrm{ZnO}: \mathrm{Al}$ front contact was deposited by magnetron sputtering. The $\mathrm{ZnO}$-bi-layer consisted of $150 \mathrm{~nm}$ 
thick intrinsic $\mathrm{ZnO}$ and $230 \mathrm{~nm}$ thick $\mathrm{ZnO}$ doped with $2 \mathrm{wt} \% \mathrm{Al}_{2} \mathrm{O}_{3}$. For electrical characterization small regions consisting of $\sim 100$ micro solar cells were separated by mechanical scribing and a $\mathrm{Ni} / \mathrm{Al}$ point contact was deposited onto $\mathrm{ZnO}$ :Al for better collection of carriers.

Reference absorbers were prepared based on a copper layer of $244 \mathrm{~nm}$ and an indium layer of $539 \mathrm{~nm}$, corresponding to a $\mathrm{Cu} / \mathrm{In}$ molar ratio of 1 . However, the precursor metals indium and copper were deposited at room temperature to avoid the formation of islands. The selenisation was performed as described before followed by the deposition of the front contact.

\subsection{Characterization techniques}

Scanning electron microscopy (SEM) and energy-dispersive X-ray (EDX) measurements were performed by a LEO GEMINI 1530 using an electron energy of $10 \mathrm{keV}$. X-ray diffraction (XRD) measurements were carried out using a D8 Advance diffractometer with $\mathrm{Cu}-\mathrm{K} \alpha_{1}$ radiation $(\lambda=1.5406 \AA)$. The analyses were performed in Bragg-Brentano diffraction conditions in the $2 \Theta$ range from $25^{\circ}$ to $35^{\circ}$ with a step size of $0.02^{\circ}$ and a step time of $2 \mathrm{~s}$. Height profiles were measured by a DEKTAK 8 profilometer from VEECO. For the room temperature photoluminescence (PL) measurements a pulsed laser diode $(655 \mathrm{~nm}$ wavelength, 2.5 MHz pulse repetition frequency) was used as optical excitation source. The PL signals were detected by a $0.5 \mathrm{~m}$ Czerny-Turner grating monochromator $(1501 / \mathrm{mm}$, spectral resolution $\sim 0.52 \mathrm{~nm}$ ) equipped with a liquid $\mathrm{N}_{2}$ cooled linear InGaAs diode array. Spatial maps of the PL signal were recorded for an exposure time of $20 \mathrm{~s}$, a $2 \mu \mathrm{m}$ step size and an optical excitation spot diameter of $\sim 1.5 \mu \mathrm{m}$. Electrical $I-V$-characterization of the micro solar cells was carried out with a classAAA solar simulator under AM1.5 standard illumination conditions.
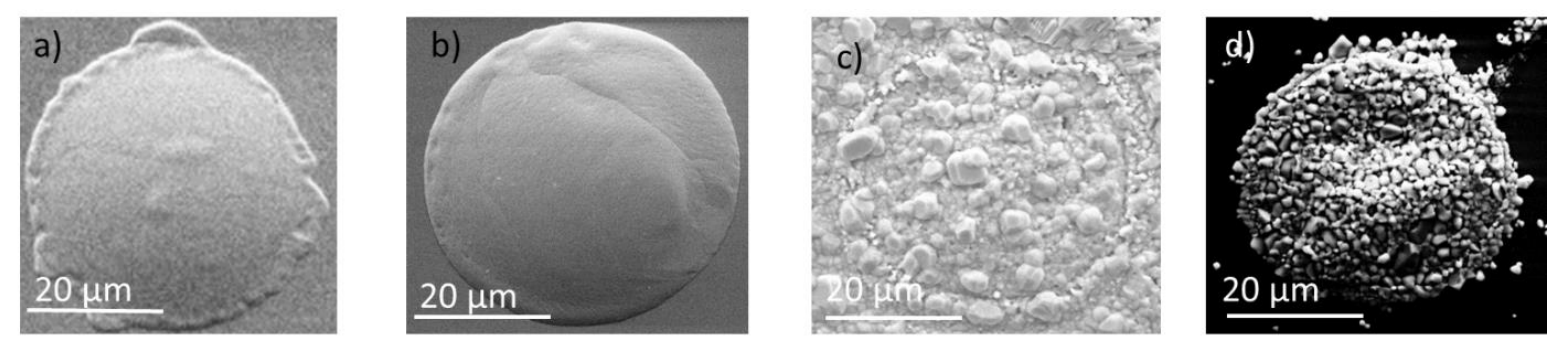

Figure 3: SEM images exemplifying the bottom-up process for CISe micro-absorber preparation. a) Bare indium island on a molybdenum layer, b) indium island coated with a $500 \mathrm{~nm}$ thick copper layer, c) sample after selenization process step, d) CISe absorber island after KCN etching.

\section{Results and discussion}

\subsection{Fabrication of micro-absorbers}


Micro-absorbers were manufactured in a bottom-up approach based on four different steps: (1) the deposition of well-controlled indium islands; (2) the deposition of a copper layer; (3) a subsequent selenization, and (4) selective removal of copper selenides. Figure 2 exemplifies the different stages of the bottom-up processing of the CISe micro-absorbers along that process chain. Indium islands of about $40 \mu \mathrm{m}$ diameter were used as precursors (Figure 3a). Figure $3 \mathrm{~b}$ demonstrates, that the islands maintain a similar morphology upon overcoating with a copper layer of $500 \mathrm{~nm}$ thickness. After selenization (Figure 3c), the surface topography is significantly roughened and copper selenides are present across the entire sample as evidenced by EDX (not shown). Subsequent chemical etching in KCN solution selectively removes the copper selenides, leaving only crystalline CISe micro-absorbers remaining on the sample surface (Figure 3d).

\subsection{Morphological characterization}

The final absorber morphology strongly depends on the copper-indium ratio and the size of the indium precursor islands. This is evidenced by a series of micro-absorbers shown in Figure 4. The upper row displays SEM images of micro-absorbers obtained from large islands of $60 \mu \mathrm{m}$ diameter (height of $\sim 4 \mu \mathrm{m}$ ), coated with copper layers of two different thicknesses, i.e. $150 \mathrm{~nm}$ (Figure $4 \mathrm{a}$ ) and $500 \mathrm{~nm}$ (Figure 4b). The lower row visualizes micro-absorbers
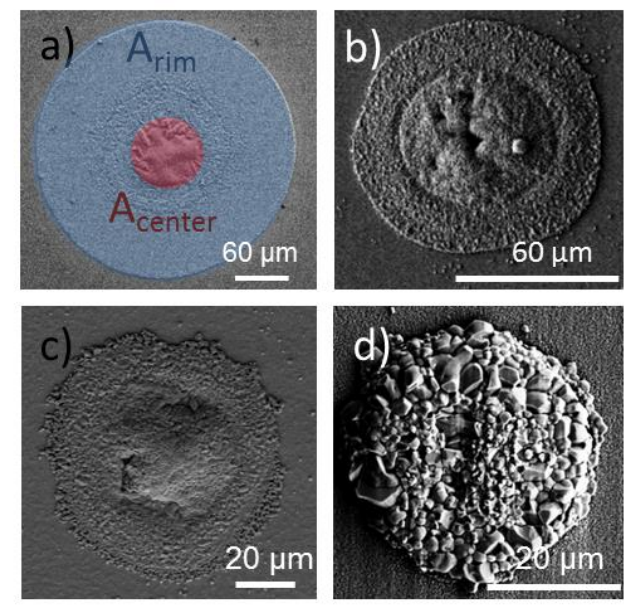

Figure 4: SEM images of micro-absorbers produced from indium islands of: a) $60 \mu \mathrm{m}$ in diameter processed with a $150 \mathrm{~nm}$ layer of copper and b) a $500 \mathrm{~nm}$ layer of copper, c) $40 \mu \mathrm{m}$ in diameter covered with a $150 \mathrm{~nm}$ layer of copper and d) a $500 \mathrm{~nm}$ layer of copper. Note the different magnifications used in the SEM images a) to d).

originating from smaller islands of $40 \mu \mathrm{m}$ diameter (height of $\sim 2 \mu \mathrm{m}$ ), which were covered with copper films of $150 \mathrm{~nm}$, (Figure 4c) and $500 \mathrm{~nm}$ (Figure 4d) thickness.

After deposition of copper, the islands had maintained their original shape as exemplarily shown in Figure 3b. After selenization, however, precursor material has spread out of the 
islands to an extent depending on the amount of copper. This effect is strongest in the case of a large indium island diameter covered by a thin copper layer (Figure 4a) and smallest in the opposite case of a small island diameter and a thick copper layer (Figure 4d). Besides the degree of spreading, the absorbers exhibit different degrees of crystallinity. The samples comprising the $150 \mathrm{~nm}$ copper layer exhibit much smaller grains $(<1 \mu \mathrm{m})$ than those prepared with a $500 \mathrm{~nm}$ copper layer $(2-3 \mu \mathrm{m})$.

By changing the thickness of the copper layer, the molar copper to indium ratio of the precursor is varied. We derived the molar ratios from the volumes of copper and indium contained in the $\mathrm{Cu}$-In precursor islands. The volumes were estimated by the model of a homogeneously thick copper layer covering an indium island, whose shape was approximated as a spherical cap. The height and diameter of the islands were determined by profilometric measurements. The molar $\mathrm{Cu}$ :In ratios corresponding to Figures $4 \mathrm{a}-\mathrm{d}$ are 0.17, 0.56, 0.33 and 1.11 , respectively. With respect to $\mathrm{CuInSe}_{2}$, with a Cu:In stoichiometry of $1: 1$, Figures $4 \mathrm{a}-4 \mathrm{c}$ show copper-deficient precursors, whereas for the sample in Figure 4d, a slight excess of copper was provided.

After material spreading, the island's central part is surrounded by an annulus, which

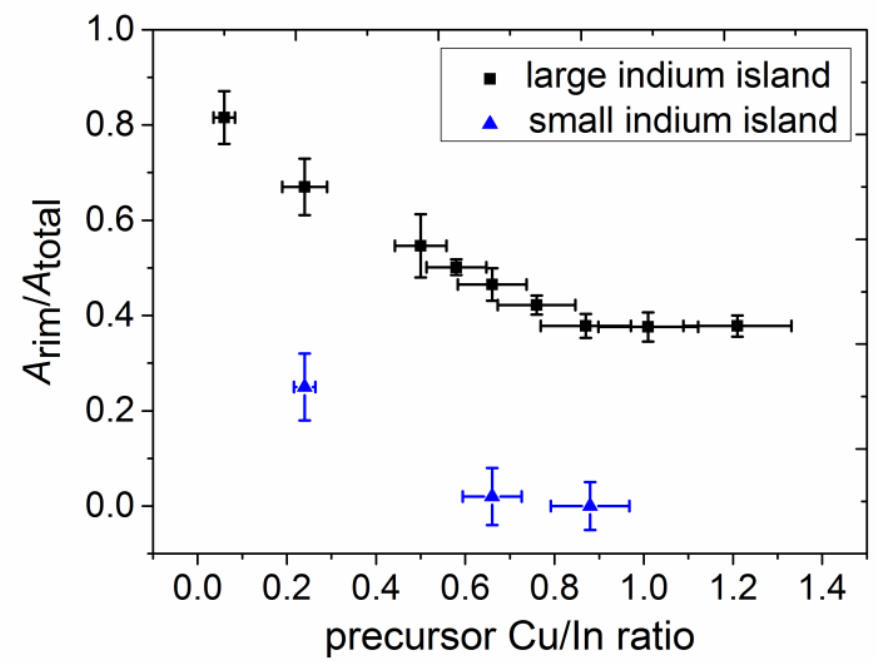

Figure 5: Ratio of $A_{\text {rim }}$ to $A_{\text {total }}$ as a function of the precursor $\mathrm{Cu} / \mathrm{In}$ ratio for large (60 $\mu \mathrm{m}$ diameter) and small indium precursor islands (40 $\mathrm{\mu m}$ diameter).

contains an area marked in Figure 4a. In the following, we will refer to the substrate

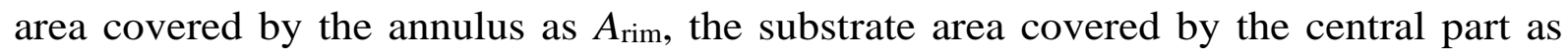
$A_{\text {central, }}$ and the substrate area covered by the sum of both as $A_{\text {total }} . A_{\text {central }}$ is coinciding with the area covered initially by the indium island and does not change for different copper film thicknesses. For indium islands of $60 \mu \mathrm{m}$ diameter, 12 different $\mathrm{Cu}$ films were tested, with thicknesses ranging from $40 \mu \mathrm{m}$ to $1000 \mu \mathrm{m}$. Additionally, for $40 \mu \mathrm{m}$ 
diameter indium islands, three different $\mathrm{Cu}$ film thicknesses were deposited (150, 400, and $500 \mu \mathrm{m}$ ). As a measure of spreading, the ratio of $\mathrm{A}_{\text {rim }}$ to $A_{\text {total }}$ is plotted with black squares as a function of the $\mathrm{Cu} / \mathrm{In}$ ratio from 0.06 to 1.2 for the $60 \mu \mathrm{m}$ indium precursors shown in Figure 5. The error bars in $y$-direction represent the standard deviation obtained from 20 different absorber islands, while the error bars in $x$ direction represent the uncertainty of the $\mathrm{Cu} / \mathrm{In}$ ratio estimation.

$A_{\text {rim }} / A_{\text {total }}$ is 0.8 for the $\mathrm{Cu}: \mathrm{In}$ ratio of 0.06 (40 nm thick $\mathrm{Cu}$ layer) and decreases continuously down to 0.38 for a $\mathrm{Cu}: \mathrm{In}$ ratio of 0.87 (500 $\mathrm{nm} \mathrm{Cu}$ layer), where it starts to saturate. The same tendency is observed for small indium islands of $40 \mu \mathrm{m}$ diameter (blue triangles). Here, the ratio of $A_{\text {rim }}$ to $A_{\text {total }}$ ranges from $0.25(150 \mathrm{~nm} \mathrm{Cu}$ layer corresponding to the $\mathrm{Cu}: \mathrm{In}$ ratio of 0.24$)$ to $0(500 \mathrm{~nm} \mathrm{Cu}$ layer corresponding to the $\mathrm{Cu}: \mathrm{In}$ ratio of 0.88 ), i.e. for a given copper layer thickness, the spreading is lower for small indium islands compared to large islands.

We assume that the degree of superficial spreading depends on the initial molar Cu:In ratio of the localized precursors. One possible explanation can be found in the alloying step taking place at $200^{\circ} \mathrm{C}$ : According to the $\mathrm{Cu}$-In phase diagram, a liquid $\mathrm{Cu}$-In alloy is in equilibrium with a solid $\mathrm{Cu}_{11} \mathrm{In}_{9}$ phase [15]. The $\mathrm{Cu}$-In alloy contains mainly indium and is saturated with about $5 \%$ of copper, indicating that a sufficiently thin outer copper layer can be dissolved completely in the liquid indium core. The liquid $\mathrm{Cu}$-In then starts to spread on the surrounding substrate covered with additional copper. After passing a certain copper volume, the alloy saturates with copper, such that solid $\mathrm{Cu}$-In phases are formed. These considerations are in line with the experimental observation, i.e. a decrease of spreading with increasing copper film thickness (see Figure 4b).

\subsection{Structural and compositional characterization}


Besides the morphological characterization by SEM, X-ray diffractograms were measured to identify the phases, which can be assigned to each absorber morphology and preparation condition. These measurements were performed after chemical etching in $\mathrm{KCN}$ solution for prior removal of $\mathrm{CuSe}_{\mathrm{x}}$ phases from the CISe absorbers. X-ray diffractograms were analyzed by comparing the diffraction maxima to the reported ones for known phases using the Crystallography Open Database (COD). Figure 6a summarizes X-ray diffractograms of absorber islands produced using various $\mathrm{Cu}$ layer thicknesses. The data were obtained after the complete precursor processing (corresponding to Figure 3d) from samples with $150 \mathrm{~nm}$ and $500 \mathrm{~nm}$ copper layer thickness on indium islands having a diameter of either $\sim 60 \mu \mathrm{m}$ or $\sim 40 \mu \mathrm{m}$. A diffractogram of a planar absorber with a stoichiometric $\mathrm{Cu}$ :In ratio is added for reference. At $2 \Theta=26.6^{\circ}$, a strong CISe (112) orientation peak appears (COD 4105347). Such an orientation of the CISe crystallite grains has been reported for chalcopyrite absorbers which is most stable in chalcopyrites [17]. Another strong peak, which can arise from the presence of $\operatorname{In}_{6} \mathrm{Se}_{7}$ and $\operatorname{In}_{2} \mathrm{Se}_{3}$ phases (COD 8104059 and COD 1528774), appears at
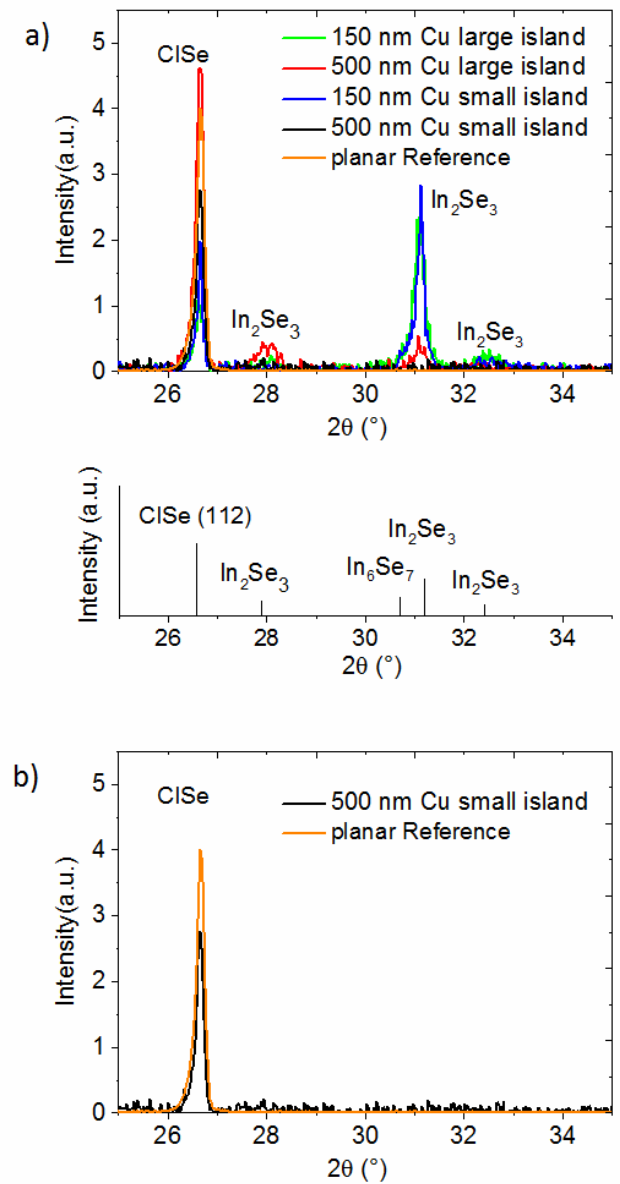

Figure 6: a) X-ray diffractograms of CISe micro-absorbers fabricated from $150 \mathrm{~nm}$ and $500 \mathrm{~nm}$ copper layers on large indium precursor islands of $\sim 60 \mu \mathrm{m}$ diameter and from $150 \mathrm{~nm}$ and $500 \mathrm{~nm}$ copper layers on small indium islands of $\sim 40 \mu \mathrm{m}$ diameter and of a planar reference absorber with positions of the reference peaks. b) Comparison of planar and micro absorber. 
$2 \Theta=31.2^{\circ}$ (except for small indium islands covered with $500 \mathrm{~nm}$ copper) and indicates, that in addition to CISe, copper free indium selenide phases have formed.

The two indium selenide phases are, however, observed for samples of molar Cu:In ratios below one. When copper is provided in excess, as in the case of small indium islands $(40 \mu \mathrm{m}$ diameter) covered with a $500 \mathrm{~nm}$ layer of copper and yielding a $\mathrm{Cu}: \mathrm{In}$ ratio of 1.1, the entire amount of indium is incorporated in CISe (black line in Figure 6a). The CISe (112) peak located at $26.6^{\circ}$ can be found for all samples, which indicates a nearly stoichiometric CISe phase with a $\mathrm{Cu}$-In ratio of 0.98 [18]. For better visibility, Figure 6b directly compares X-ray diffractograms obtained from a planar reference (orange curve) with that from an arrangement of several $40 \mu \mathrm{m}$ absorbers (black curve) providing a similar Cu:In ratio ( 1). Apparently, even in the copper-rich sample (black curve in Figure 6b), a small fraction of copper is not included in the absorber, but is instead converted to copper selenides, which have been etched off before the XRD measurements. From the combined morphological and phase analysis we conclude, that in our set of samples, least spreading and the highest purity of the CISe phase are observed for small precursor islands ( $40 \mu \mathrm{m}$ diameter) with a slight excess of copper (500 $\mathrm{nm}$ copper film). The X-ray diffractogram of this sample is in excellent agreement with the planar reference absorber (Figure 6b). Complementary micro Raman scattering measurements on the micro absorbers are characterized by the dominant $\mathrm{A}_{1}$-mode of CISe and reveal the absence of ordered defect compounds for depths up to about $100 \mathrm{~nm}$ below the surface (data not shown here). Hence, we assume that the XRD signal at $26.6^{\circ}$ shown in Figure 6 originate solely from polycrystalline CISe material. For further investigation of topography and lateral and vertical compositional profiles of the micro-absorbers, a large (60 $\mu \mathrm{m}$ diameter, Figure 7$)$ and a small (40 $\mu \mathrm{m}$ diameter, Figure 8$)$ micro-absorber processed with $500 \mathrm{~nm}$ copper layers were characterized by profilometry as well as top-view and cross-sectional SEM/EDX. 

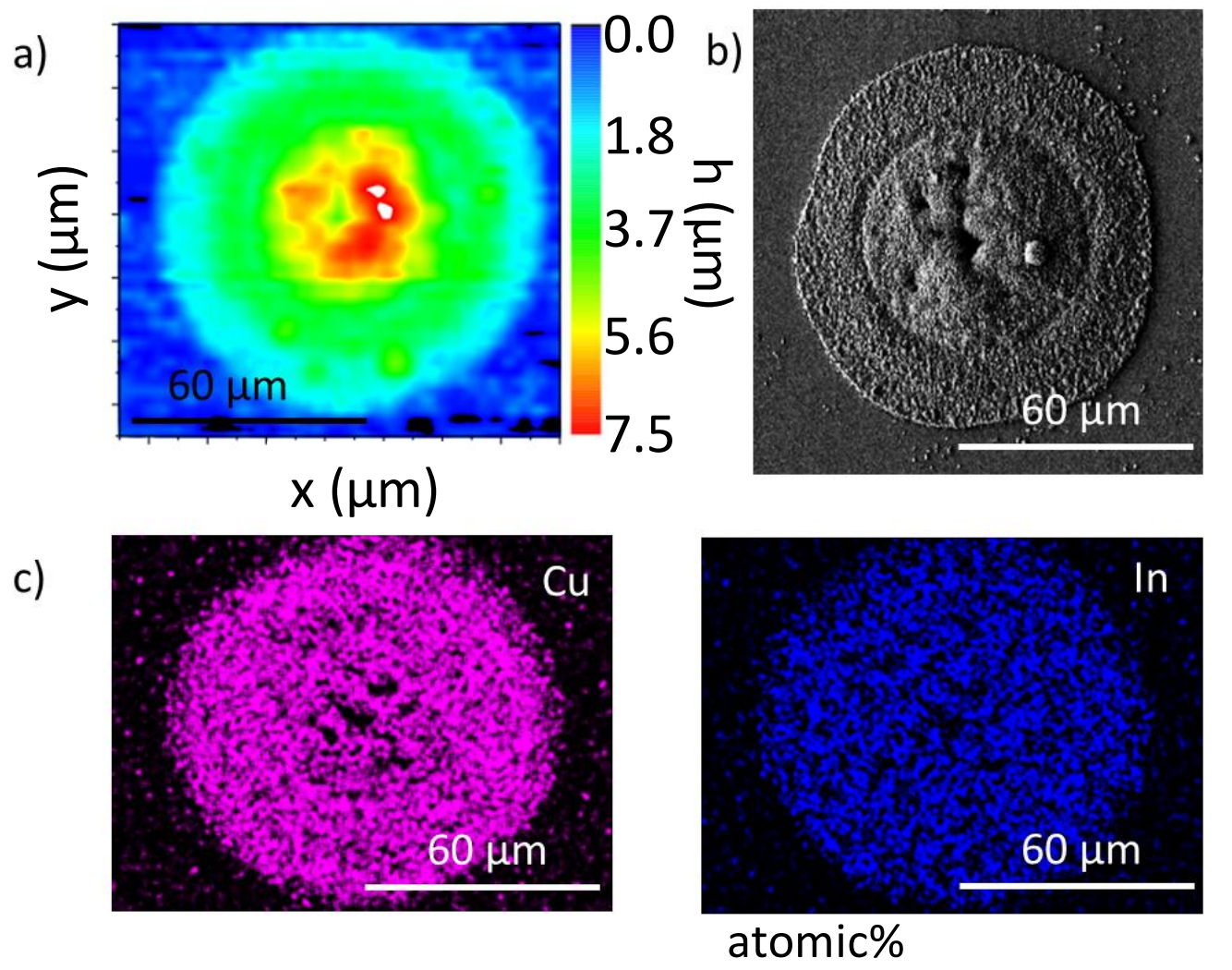

d)

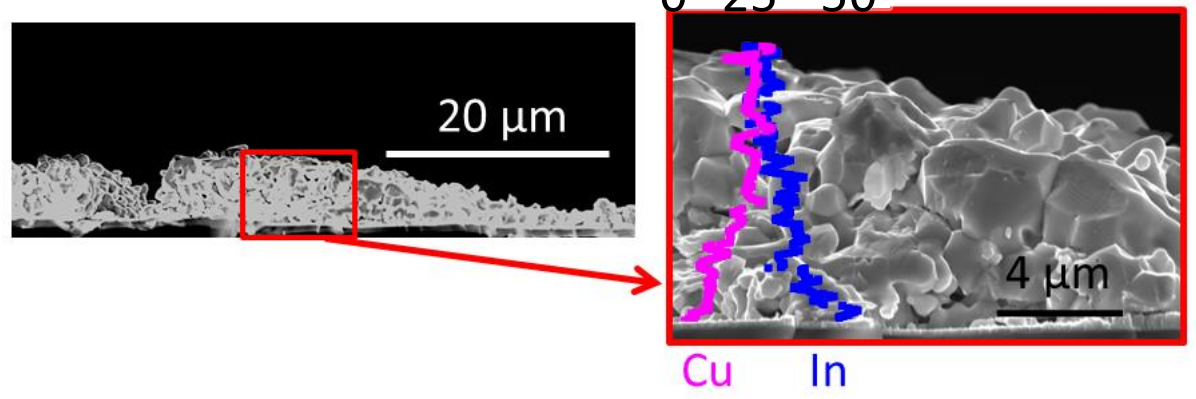

Figure 7: Micro-absorber produced from an indium island with an initial diameter of approximately $60 \mu \mathrm{m}$ and a copper layer of $500 \mathrm{~nm}$. a) Profilometric topography, b) top-view SEM image, c) maps of atomic weight copper counts and indium counts measured by EDX in top view, d) SEM of micro-absorber cross section and vertical EDX line scan (indium and copper signals).

For large micro-absorber islands, the height increases gradually from the edge towards the center, starting with approximately $1.8 \mu \mathrm{m}$ at the rim and reaching a maximum height of approximately $7.5 \mu \mathrm{m}$ at the center (Figure 7a).The total diameter of those absorbers is around $75 \mu \mathrm{m}$ with a core diameter of $60 \mu \mathrm{m}$ and a rim of $15 \mu \mathrm{m}$ (see discussion of Figure 4). The height of the absorber has increased by a factor of about 2 during selenization. As can be observed both from the profilometric measurement (Figure 7a) and the SEM images (Figure $7 \mathrm{~b}$ and $7 \mathrm{~d}$ ), a small cavity is present in the center of this micro-absorber. Given the influence of the surface topography, the corresponding EDX mapping of the elemental distributions of $\mathrm{Cu}$ and $\mathrm{In}$ (Figure 7c) indicate a widely homogeneous lateral distribution of 
copper and indium over the escape length of X-rays in EDX, which is typically less than $1 \mu \mathrm{m}$ (for L-line signals) [19]. The surface roughness is a material property resulting from the fabrication process. As opposed to the surface EDX mapping the vertical cross-sectional EDX line scans (Figure 7d) shows a concentration gradient for both indium and copper. At the top, a stoichiometric ratio is observed with atomic percentages of $25 \%$ for both elements. Towards the molybdenum layer, however, the atomic percentage of copper decreases while the atomic percentage of indium increases. The missing of $50 \%$ of atomic percentage are attributed to selenium incorporated inside the absorber (selenium signal not shown here). The crosssectional EDX line scan is performed near the center of the micro-absorber (Figure 7d), see the red squared part marked on the left SEM image where the absorber has a height of approximately $7 \mu \mathrm{m}$. The distribution of copper and indium indicates that the indium selenide phases of this sample detected by XRD (Figure 6) are located in the inner core of the island, where the intermixing of copper and indium seems to be incomplete in the case of large indium islands. Moreover, the cross-sectional EDX line scan (Figure 7d) and the XRD measurement (Figure 6) are fully consistent. Supposedly, the deviation of the indium and copper concentration in the micro absorber from the ideal conditions (1:1) results in band gap variations. In order to avoid this detrimental effect in our specific case smaller indium islands may be beneficial. 


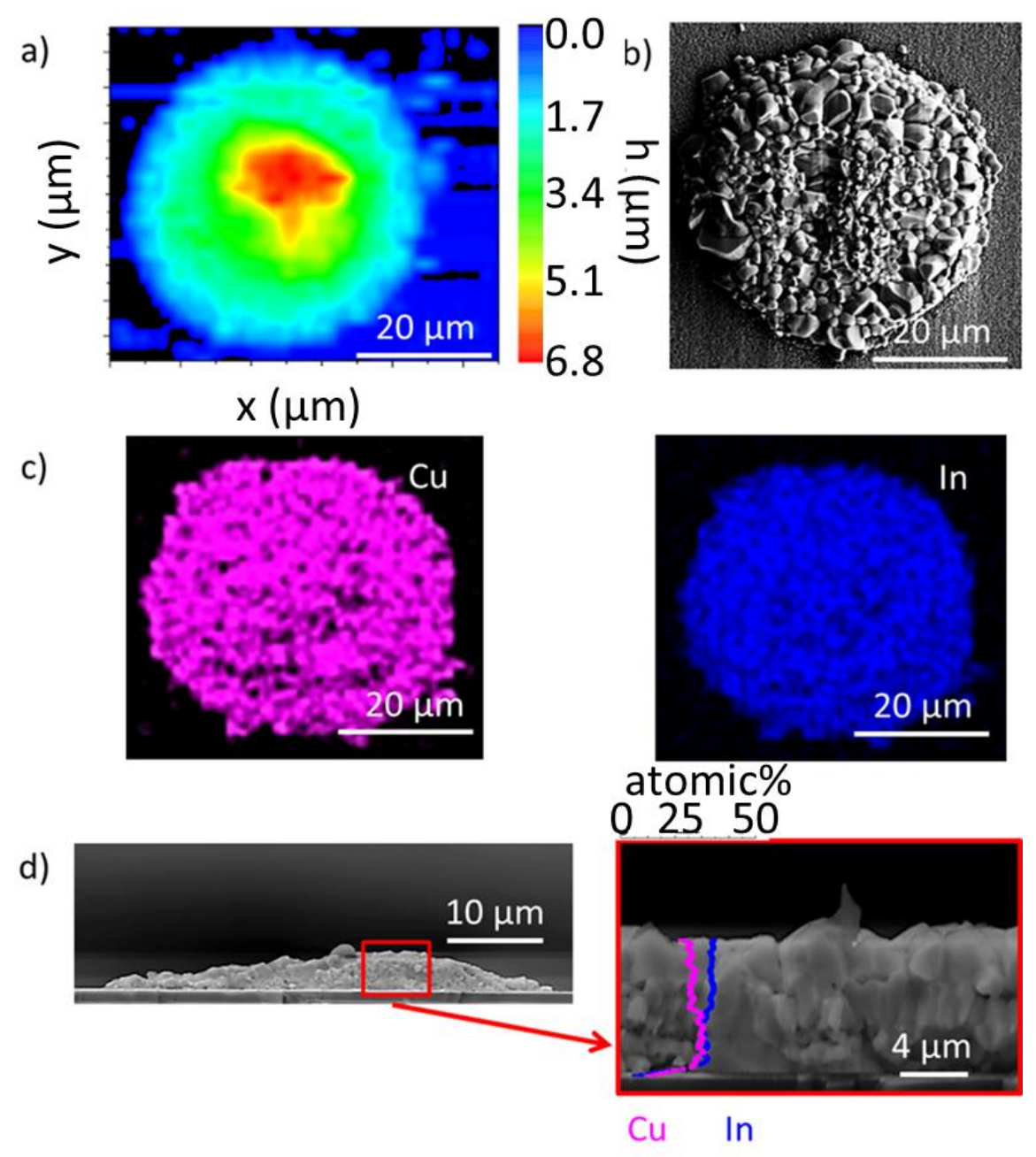

Figure 8: Micro-absorber produced from an indium island with an initial diameter of approximately $40 \mu \mathrm{m}$ and a copper layer of $500 \mathrm{~nm}$ thickness. a) Profilometric measurement, b) SEM image, c) map of copper and indium counts measured by EDX in top view, d) SEM cross section and vertical EDX line scan (indium and copper signals).

For small micro-absorber islands, the profilometric measurement also shows a gradual height increase towards the center (Figure 8a). The corresponding SEM micrograph is shown in Figure $8 \mathrm{~b}$. The minimum height at the rim is $1.7 \mu \mathrm{m}$ and the maximum height in the center $6.8 \mu \mathrm{m}$. The total absorber diameter is about $40 \mu \mathrm{m}$. An outer rim and a cavity in the center are absent for this kind of absorbers. In brief, the small islands have not extended in the horizontal direction after selenization, but the height has increased by a factor of about 3 . Such an increase in thickness is also commonly observed for CISe films upon the incorporation of selenium and phase transformation. The top-view EDX mapping gives the same result as for the large islands, i.e. indium and copper are homogenously distributed (Figure 8c). In contrast to the large absorbers of $60 \mu \mathrm{m}$ diameter, according to the vertical cross-sectional EDX line scan, the distribution of copper and indium between top and bottom 
of the island is nearly uniform (Figure 8d). There is a slightly copper-depleted Cu:In ratio at the CISe surface and a nearly stoichiometric $\mathrm{Cu}$ :In ratio at the CISe-molybdenum interface. The cross-sectional EDX was performed in the red marked part of Figure 8d. Apparently, the intermixing of indium and copper during the alloying phase is more efficient for small indium islands, where shorter diffusion paths have to be covered due to a smaller indium island diameter and thickness. Since the copper layer was covering the indium island from all sides the diffusion was expected to be not only in thickness direction, but (to a minor extent) also in

a)

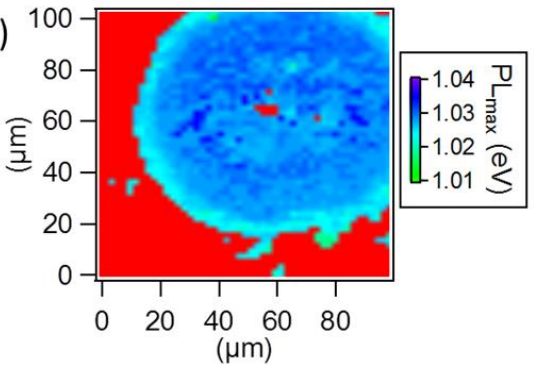

d)

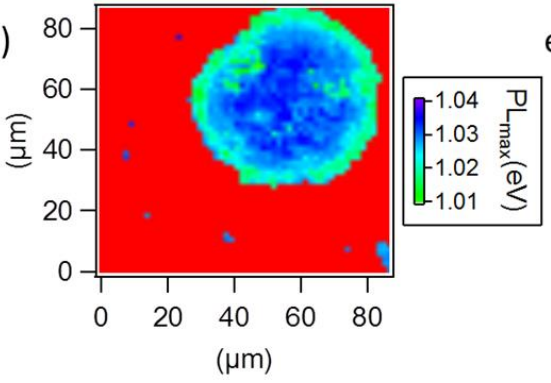

g)

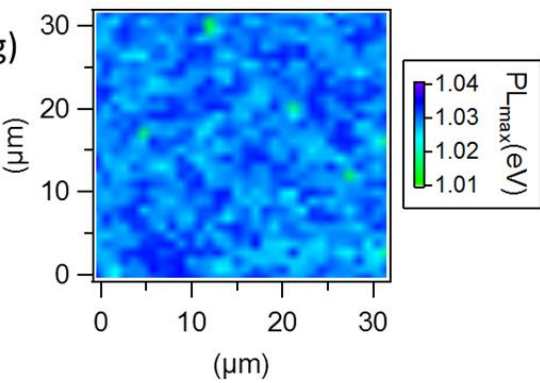

b)

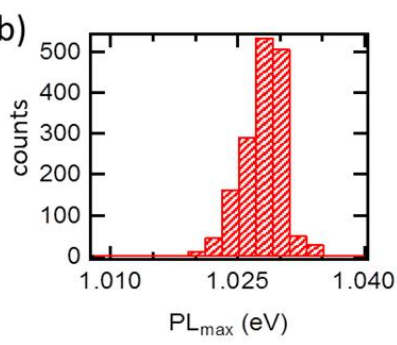

e) $200+\frac{1}{1}, 1,0$

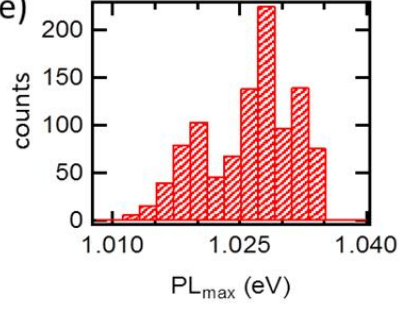

h)

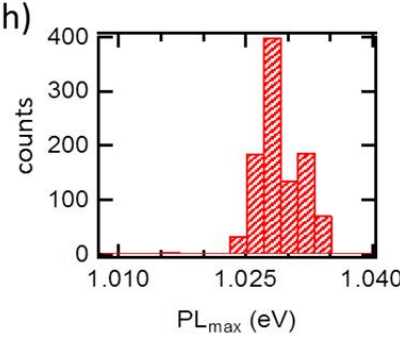

C)
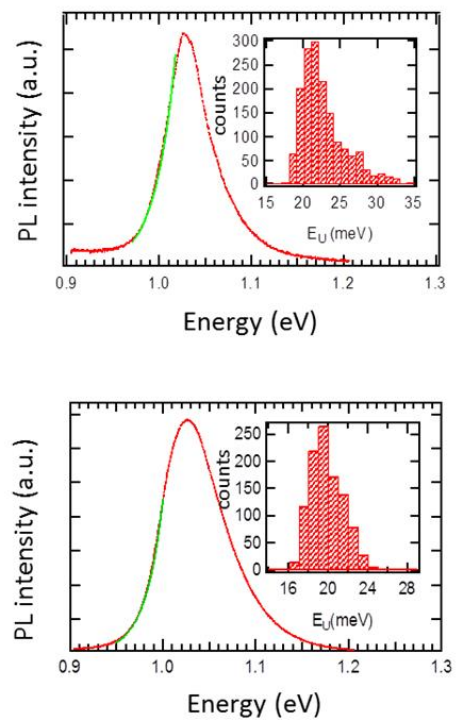

i)

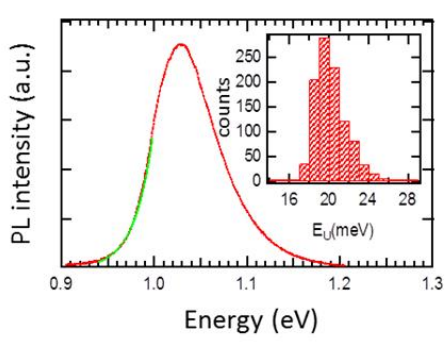

Figure 9: a), d) and g) PL map of peak emission energies; b), e) and h) histogram of peak emission energy; c), f) and i)

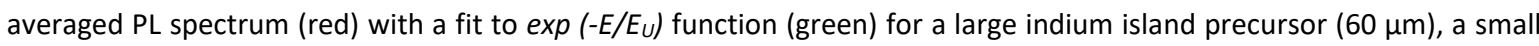
indium island precursor $(40 \mu \mathrm{m})$ and a planar $\mathrm{CISe}$ absorber. Inset in $\mathrm{c}), \mathrm{f})$ and i): histogram of $E_{U}$ characteristic energy.

horizontal direction from the island sides. A shorter diffusion length leads to a more uniform composition and surface topography which is highly desirable for micro-concentrator solar cell applications.

\subsection{Optical characterization}

For investigating the optoelectronic properties of the micro-absorbers, photoluminescence mapping measurements were performed with a spatial resolution and step size of about $2 \mu \mathrm{m}$. 
As a reference, the same measurement was carried out on a planar CISe absorber. A map of the spectral PL peak emission position is shown in Figure 9a, 9d and 9g for a large indium island precursor $(60 \mu \mathrm{m})$, a small indium island precursor $(40 \mu \mathrm{m})$ and a planar CISe absorber, where the green color represents a lower energy at about $1.01 \mathrm{eV}$, the purple color a higher energy peak position at about $1.04 \mathrm{eV}$, the red color the molybdenum substrate. Statistical analysis of those PL maps yields a homogeneous distribution of the PL maximum position across the investigated sample area (Figure 9b, 9e and 9h). Comparison of the full width at half maximum (FWHM) of the PL spectra showed a good agreement between the small island and the planar absorber (FWHM $\sim 80 \mathrm{meV}$ ), while the FWHM of the large island was found to be narrower by $20 \mathrm{meV}$.

The PL spectra of the CISe islands were averaged over all measured points and the resulting spectrum is plotted as a function of energy in Figure 9c, 9f and 9i for the large island, the small island and the planar absorber. Small variations in PL maximum in micro meter spots were possibly caused by small changes in $\mathrm{Cu}$ content [20]. We have examined the high energy side of the PL transition with a model that assumes the Boltzmann distribution for the occupation of carriers and square root function for the absorption coefficient. By neglecting the energy dependence of the transition matrix element this model gives [21]

$I_{P L}(E) \propto E^{2}\left(E-E_{g}\right)^{0,5} \exp (-E / k T)$,

where $E$ is the photon energy, $E_{G}$ the optical band gap, $k$ the Boltzmann constant, $T$ the carrier temperature, and the $E^{2}$ term arises from the photon density of states. An analysis of the high energy PL side (not shown here) with this model results in $T$ values in the range of $300 \mathrm{~K}$ $320 \mathrm{~K}$, corresponding well to the actual measurement ambient temperature and confirming the model.

In order to interpret the low energy PL tail we considered an exponential density of states for photon energies smaller than $E_{G}$. Assuming full occupation of the tail states $I_{P L}(E)$ was fitted to $\exp \left(-E / E_{U}\right)$ function, where $E_{U}$ is the characteristic energy. As seen from Figure 9c, 9f and 9i we obtain a good agreement between the PL data (red curves) and the exponential fit (green curves) yielding $E_{U} \approx 20 \mathrm{meV}$ for all samples. The corresponding histogram plots of $E_{U}$, obtained from the entire absorber, are also presented in the inset of Figure 9c, 9f and 9i, indicating that this value is a characteristic parameter for the complete CISe samples. Note that usually higher values of $E_{U}$ of about $20 \mathrm{meV}-60 \mathrm{meV}$ are reported in Cu-poor CISe samples [22]. In general, the smaller the $\mathrm{E}_{\mathrm{U}}$ parameter, the less tail states are present in the forbidden band gap. 
The band-to-band recombination is the process with the highest emission energy and dominates the CISe luminescence at room temperature. Therefore, the energy of the band-toband recombination can be associated with the optical band gap energy. Comparing this transition energy for the large micro island, the small micro island and the planar absorber shows perfect agreement with a transition energy of $1.03 \pm 0.01 \mathrm{eV}$ determined for all samples. Similar PL spectra and band gaps were also reported for planar stoichiometric CISe absorbers in the literature, whereas $\mathrm{Cu}$-poor samples were found to show transition energies significantly below $1 \mathrm{eV}[21,23]$.

\subsection{Electrical characterization}

Electrical characterization was performed by $I-V$ curve measurement via a solar simulator under AM1.5 standard one sun illumination (red curve) and a dark measurement (black curve) (Figure 10). A mechanically scribed area of $0.3 \times 0.3 \mathrm{~cm}^{2}$ with around 100 microsolar cells, fabricated from the optimized absorbers, was measured (inset of Figure 10). For better collection of carriers during the $I-V$ characterization, a Ni:Al front contact was deposited in

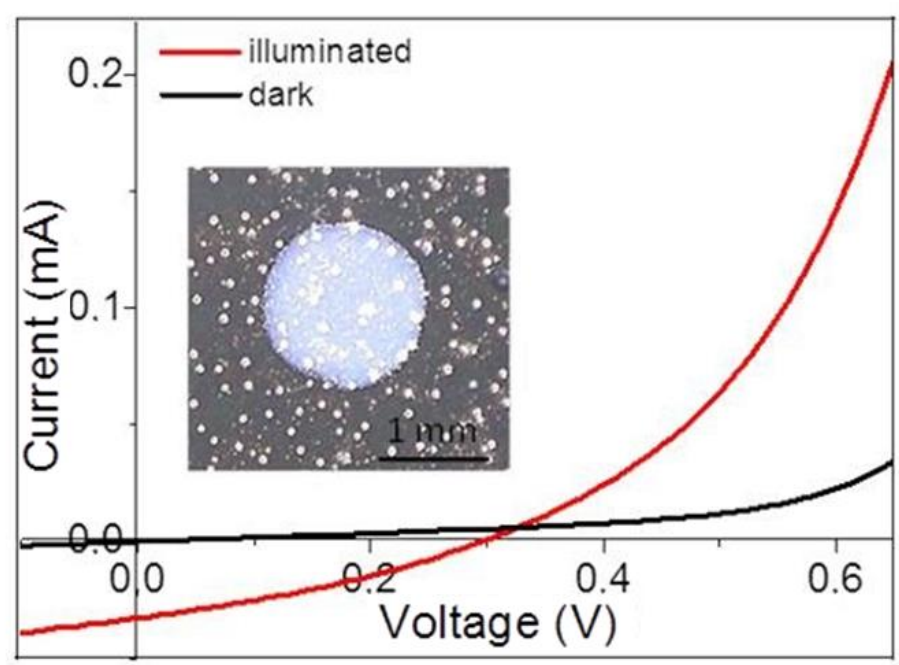

Figure 10: IV measurement of micro solar cells (dark curve in black and illuminated curve in red). Inset: A microscopy image of the measured sample area including about 100 micro absorbers (white dots). The Ni/Al contact is visible as bluish point in the center of the area.

the center of the scribed area. The obtained $I-V$ curve revealed a diode-like behavior with an open circuit voltage $\left(V_{O C}\right)$ of $294 \mathrm{mV}$, a short circuit current $\left(I_{S C}\right)$ of $0.033 \mathrm{~mA}$, and a fill factor $(F F)$ of $36 \%$. Assuming that all of the 100 micro-absorbers in the measured area contribute to the active absorber area, the active absorber area quantified from optical microscopy is $(0.0012 \pm 0.00007) \mathrm{cm}^{2}$. Using this estimated active area we can calculate a short circuit current density of $\left(J_{S C} \sim 27.5 \pm 2.3\right) \mathrm{mA} / \mathrm{cm}^{2}$ and a solar cell efficiency of $(2.9 \pm 0.2) \%$. The planar reference absorber of $0.5 \mathrm{~cm}^{2}$ area achieved $\sim 5.9 \%$ efficiency with a $J_{S C}$ of $33 \mathrm{~mA}$, a $V_{O C}$ of $406 \mathrm{mV}$ and a $F F$ of $44 \%$ (results not shown here). 
The micro solar cells showed around half of the efficiency of the reference solar cell. One possible reason is surface recombination that reduces the carrier densities. Recombination is caused by defects or impurities near the absorber surface or at the absorber-isolator interface and has a major impact on short-circuit current and open-circuit voltage. Moreover, the thickness variation across the micro absorbers (see Figures 7a and 8a) can affect the carrier transport, resulting in a local change of the series resistance. Hence, for larger absorber thicknesses electron-hole recombination can limit the cell efficiency here. Both, $I_{S C}$ and $V_{O C}$, will decrease with higher recombination. It should be noted that the surface-to-volume ratio is increased for an individual micro solar cell $\left(\sim 1 \times 10^{4} 1 / \mathrm{cm}\right.$; estimated on basis of the cell's active absorber area divided by its volume) when compared to the planar reference $\left(\sim 5 \times 10^{3} 1 / \mathrm{cm}\right)$. Due to this increased surface-to-volume ratio of the micro cells, surface recombination effects are more relevant than in planar devices. Furthermore, the $I-V$ characteristic (Figure 10) indicate that our cell assembly is affected by series and shunt resistances, which (in combination) reduce $I_{S C}$ and $V_{O C}$. The total series resistance accounts to $\sim 9$ (corresponding to $0.01 \Omega \mathrm{cm}^{2}$ for the active absorber area) for the assembly of $\sim 100$ micro solar cells. The planar reference cell exhibits a series resistance of approx. $2 \Omega$ (corresponding to $1 \Omega \mathrm{cm}^{2}$ for the active absorber area). The corresponding shunt resistances are $\sim 45 \mathrm{k} \Omega$ (corresponding to $54 \Omega \mathrm{cm}^{2}$ for the active absorber area ) (micro solar cells) and $\sim 50 \mathrm{k} \Omega$ (corresponding to $25 \mathrm{k} \Omega \mathrm{cm}^{2}$ for the active absorber area) (reference cell). The same trend of reduced efficiencies along with reduced $I_{S C}, V_{O C}$, and $F F$ was recently reported for electrodeposited bottom-up micro cells [12]. Nevertheless, the difference between the $I-V$ curves measured in dark and illuminated conditions clearly demonstrates the functionality of our bottom-up micro solar cell device. The electrical performance was characterized also at three suns illumination, resulting in $V_{O C}=360 \pm 7 \mathrm{mV}, I_{S C}$ $=0.102 \pm 0.01 \mathrm{~mA}, F F=37 \pm 1 \%$, and an efficiency of $3.1 \pm 0.2 \%$. Hence the cells show an increase of the efficiency under light concentration.

The solar cell performance also could be optimized later (1) by providing a Ni:Al grid between ordered micro solar cell for reducing the carrier diffusion paths to the front contact, (2) by optimizing the isolation layer in order to reduce recombination at the absorber interface, and (3) by adapting the thickness of the CdS layer for the micro solar cell device.

\section{Conclusion}

The manufacturing of CISe micro-absorbers and micro solar cells is demonstrated for the first time in a bottom-up approach, which is based on the processing of well-controlled indium 
islands, followed by the deposition of a copper layer, selenization and selective removal of copper selenides. By varying the indium island diameters from $60 \mu \mathrm{m}$ to $40 \mu \mathrm{m}$ and the thickness of the covering copper film from $150 \mathrm{~nm}$ to $500 \mathrm{~nm}$, the CISe micro-absorbers were optimized. The morphology and phase composition of the micro-absorbers both strongly depend on the initial copper-to-indium ( $\mathrm{Cu}: \mathrm{In})$ ratio of the precursor. Generally, $\mathrm{Cu}: \mathrm{In}$ ratios lower than one result in spreading of the precursor material during the selenization step. A low $\mathrm{Cu}$ :In ratio leads to an incomplete incorporation of indium in CISe and a partial formation of indium-selenides. Micro-absorbers with a pure CISe phase can be obtained by providing a slight excess of copper in the precursor. For these absorbers a homogeneous elemental distribution is realized. The resulting CISe micro-absorbers exhibit an optical band gap energy of about $1.03 \mathrm{eV}$ and a high spatial homogeneity of photoluminescence across the entire island. With the optimized micro-absorbers, solar cells can be produced, which offer diode behavior and promise an efficiency of $(2.9 \pm 0.2) \%$. Our approach is promising as it offers the benefit of material saving by using only $1 \%$ of indium compared to planar solar cells. Future work will include further optimization of the solar cell device and utilize the fs-laser technology [13] to realize an arrangement with micro concentrator optics.

\section{Acknowledgements}

The authors gratefully acknowledge financial support by the Deutsche Forschungsgemeinschaft (DFG) through BO 1129/6-1, KR 3638/3-1 and SCHM 2554/3-1. The research leading to these results has received funding from the European Union Seventh Framework Programme (FP7/2007-2013) under grant agreement $n^{\circ}$ 609788. B. Heidmann and M. Schmid are grateful to the Helmholtz Association for support from the Initiative and Networking Fund for the Young Investigator Group VH-NG-928. The authors would like to thank Dr. D. Abou-Ras for access to the SEM/EDX. The authors would like to thank M. Kirsch for $\mathrm{ZnO}$ sputtering and grid deposition as well as O. Ernst for preparation of the isolating layers.

\section{References}

[1] T. Mai, M. Hand, S. F. Baldwin, P. Denholm, M. Milligan, Renewable Electricity Futures for the United States, IEEE Transactions on sustainable energy, 5 (2014) 372-377.

[2] S. P. Philipps, A. W. Bett, K. Horowitz, S. Kurtz, Current Status of Concentrator Photovoltaic (CPV) Technology, Fraunhofer ISE, NREL, 2016; pp. 1-26.

[3] M. Paire, A. Shams, L. Lombez, N. Péré-Laperne, S. Collin, J.-L. Pelouard, J.-F. Guillemoles, D. Lincot, Resistive and thermal scale effects for $\mathrm{Cu}(\mathrm{In}, \mathrm{Ga}) \mathrm{Se} 2$ 
polycrystalline thin film microcells under concentration, Energy Environ. Science 4 (2011) 4972-4977.

[4] M.W. Haney, Can Integrated Micro-Optical Concentrator Technology Revolutionize Flat-Plate Photovoltaic Solar Energy Harvesting?, Journal of Physics: Conference Series 660 (2015) 012014.

[5] A. Marti, G. L. Araujo, Limiting efficiencies for photovoltaic energy conversion in multigap systems, Sol. Energy Mat.Sol.Cells 43(2) (1996) 203-222.

[6] J. Yoon, A. J. Baca, S. I. Park, P. Elvikis, J. B. Geddes, L. Li, R. H. Kim, J. Xiao, S. Wang, T. Ho-Kim, J. M. Motala, B. Y. Ahn, E. B. Duoss, J. A. Lewis, R. G. Nuzzo, P. M. Ferreira, Y. Huang, A. Rockett, J. A. Rogers, Ultrathin silicon solar microcells for semitransparent, mechanically flexible and microconcentrator module designs, Nature Materials 7 (2008) 907-916.

[7] M. Paire, L. Lombez, F. Donsanti, M. Jubault, S. Collin, J.-L. Pelouard, J.-F. Guillemoles, D Lincot., $\mathrm{Cu}(\mathrm{In}, \mathrm{Ga}) \mathrm{Se} 2$ microcells: High efficiency and low material consumption, Journal of Renewable and Sustainable Energy 5 (2013) 011202-1-011202-5.

[8] B. Reinhold, M. Schmid, D. Greiner, M. Schüle, D. Kieven, A. Ennaoui, M. C. Lux-Steiner, Monolithically interconnected lamellar $\mathrm{Cu}(\mathrm{In}, \mathrm{Ga}) \mathrm{Se}_{2}$ micro solar cells under full white light concentration, Progress in Photovoltaics: Research and Applications 23 (2015) 1929-1939.

[9] E. Lotter, P. Jackson, S. Paetel, W. Wischmann, Identification of Loss Mechanisms in CIGS Micro-Cells for Concentrator Applications, EU-PVSEC Proceedings 32nd European Photovoltaic Solar Energy Conference and Exhibition, Munich, 2016, 1156-1160.

[10] S. Jutteau, J.-F. Guillemoles, M. Paire, Study of a micro-concentrated photovoltaic system based on $\mathrm{Cu}(\mathrm{In}, \mathrm{Ga}) \mathrm{Se} 2$ microcells array, Applied Optics 55 (2016) 6656-6661.

[11] S. Sadewasser, P. M. P. Salome, H. Rodriguez-Alvarez, Materials efficient deposition and heat management of CuInSe2 micro-concentrator solar cells, Sol. Energy Mat. Sol. Cells 159 (2017) 496-502.

[12] A. Duchatelet, K. Nguyen, P.-P. Grand, D. Linot, M. Paire, Self-aligned growth of thin film $\mathrm{Cu}(\mathrm{In}, \mathrm{Ga}) \mathrm{Se} 2$ solar cells on various micropatterns, Applied Physics Letters 109 (2016) 253901 
[13] F. Ringleb, K. Eylers, T. Teubner, T. Boeck, C. Symietz, J. Bonse, S. Andree, J. Krüger, B. Heidmann, M. Schmid, M. Lux-Steiner, Regularly arranged indium islands on glass/molybdenum substrates upon femtosecond laser and physical vapor deposition processing, Applied Physics Letters 108 (2016) 111904.

[14] F. Ringleb, K. Eylers, T. Teubner, H.-P. Schramm, C. Symietz, J. Bonse, S. Andree, B. Heidmann, M. Schmid, J. Krüger, T Boeck. Growth and shape of indium islands on molybdenum at micro-roughened spots created by femtosecond laser pulses, Applied Surface Science 418 (2017) 548-553.

[15] P.R. Subramanian, D.E. Laughlin, The Cu-In (Copper-Indium) System, Bulletin of Alloy Phase Diagrams 10 (1989) 554-568.

[16] MicroChem, SU-8 Permanent Photoresist.

http://www.microchem.com/pdf/SU-8-table-of-properties.pdf, 2016 (accessed: 03.12.2016.

[17] Siebentritt S., Papathanasiou N., Albert J., Lux-Steiner M. Ch., Stability of surfaces in the chalcopyrite system. Applied Physics Letters 2006; 88: 151919-1 151919-3.

[18] http://www.crystallography.net/cod/, Crystallography Open Database, accessed: December, 2016

[19] D. Abou-Ras, R. Caballero, C. H. Fischer, C. A. Kaufmann, I. Lauermann, R. Mainz, H. Mönig, A. Schopke, C. Stephan, C. Streeck, S. Schorr, A. Eicke, M.

Dobeli, B. Gade, J. Hinrichs, T. Nunney, H. Dijkstra, V. Hoffmann, D. Klemm, V. Efimova, A. Bergmaier, G. Dollinger, T. Wirth, W. Unger, A. A. Rockett, A. Perez-Rodriguez, J. Alvarez-Garcia, V. Izquierdo-Roca, T. Schmid, P. P. Choi, M. Müller, F Bertram., J. Christen, H. Khatri, R. W. Collins, S. Marsillac, I. Kotschau, Comprehensive Comparison of Various Techniques for the Analysis of Elemental Distributions in Thin Films, Microscopy and Microanalysis 17 (2011) 728-751.

[20] L. Gütay, D. Regesch, J. K. Larsen, Y. Aida, V. Depredurand, A. Redinger, S. Caneva, S. Schorr, C. Stephan, J. Vidal, S. Botti, S. Siebentritt, Feedback mechanism for the stability of the band gap of CuInSe 2 , Phys. Rev. B 86 (2012) 045216-1-045216-5.

[21] T. Unold, L. Gütay, Photoluminescence Analysis of Thin-Film Solar Cells, in U. Rau, D. Abou-Ras, T. Kirchartz (Eds.), Advanced Characterization Techniques for Thin Film Solar Cells, Wiley-VCH: Weinheim, Germany, 2011; pp. 151. 
[22] S. Siebentritt, N. Papathanasiou, M.Ch. Lux-Steiner, Potential fluctuations in compensated chalcopyrites, Physica B 376-377 (2006) 831-833.

[23] S. Chichibu, Room-temperature near-band-edge photoluminescence from $\mathrm{CuInSe}_{2}$ heteroepitaxial layers grown by metalorganic vapor phase epitaxy, Applied Physics Letters 70 (1997) 1840-1842. 